\title{
The interaction of a plasmoid with a loop-top kernel
}

\author{
S. Kołomański ${ }^{1,2}$ and M. Karlický ${ }^{1}$ \\ 1 Astronomický ústav AV ČR, Fričova 298, 25165 Ondřejov, Czech Republic \\ 2 Instytut Astronomiczny Uniwersytetu Wrocławskiego, Kopernika 11, 51-622 Wrocław, Poland \\ e-mail: kolomans@astro.uni.wroc.pl
}

Received 26 July 2007 / Accepted 22 August 2007

\section{ABSTRACT}

\begin{abstract}
Aims. We study the interaction between a downward moving plasmoid and a loop-top kernel recognized in the 30 November 2000 flare. Such an interaction is predicted by some numerical models of solar flares.

Methods. Using X-ray observations from Yohkoh and GOES, EUV observations from SOHO, and radio observations from Ondřejov, we perform multi-wavelength analysis of this interaction.

Results. The Yohkoh/SXT and SOHO/EIT images indicate that the growing flare loop with the loop-top kernel and the above-lying plasmoid were formed as a result of the interaction of two extended arcade-loops. While the flare loop was growing upwards, the plasmoid moved downwards with the velocity of about $16 \mathrm{~km} \mathrm{~s}^{-1}$ and interacted with the loop-top kernel. Many details of this interaction are found, e.g., an increase of the X-ray and decimetric radio fluxes and an increase of the plasma temperature at the interaction site. Just after the coalescence of the plasmoid with the loop-top kernel, the 1-2 GHz pulsating radio structure and hard $\mathrm{X}$-ray source above the coalescence site were observed. The analyzed temperature maps indicate flows of heated plasma around the plasmoid to the location of the X-ray and radio source. These observations are in agreement with predictions from numerical modelling.
\end{abstract}

Key words. Sun: corona - Sun: flares - Sun: X-rays, gamma rays - Sun: magnetic fields - Sun: radio radiation

\section{Introduction}

The most common model of large solar flares is that in which there is an upward moving magnetic rope, below which the reconnecting current sheet, cusp structure and flare loop arcade are formed (Magara et al. 1996; Shibata 1999; Priest $\&$ Forbes 2000). There are many observations supporting this model (Tsuneta et al. 1992; Yokoyama et al. 2001; Krucker et al. 2003; Lin et al. 2003; Chen et al. 2004).

The observations of X-ray loop-top sources (loop-top kernels; e.g.: Acton et al. 1992; Masuda et al. 1994) also favour this model. Namely, as shown by Karlický \& Kosugi (2004) and Karlický \& Bárta (2006), these loop-top sources can be naturally explained by the electron acceleration in the collapsing magnetic trap, which is a part of this flare model (Somov \& Kosugi 1994).

The observation of the plasmoid ejection further supports this flare model (Ohyama \& Shibata 1998). In the papers by Kliem at al. (2000), Khan et al. (2002), and Karlický (2004), it was found that these plasmoid ejecta are associated with the drifting pulsating structures (DPS) observed in the decimetric frequency range. These DPSs have both negative and positive frequency drifts, which correspond to upward or downward motion of the plasmoids. Furthermore, there are observations (Karlický 2004) showing several simultaneous DPSs, which indicates several plasmoids. These observations agree with the concept of the fractal reconnection proposed by Shibata \& Tanuma (2001) and also with numerical simulations (Bárta et al. 2007; Karlický \& Bárta 2007), where the interaction of plasmoids are also shown. Some simulations (Bárta et al. 2007; Riley et al. 2007) even show that downward moving plasmoids interact with the flare loop arcade. Using unique observations of the
30 November 2000 flare, this process is studied in detail in this paper.

\section{Data sets}

The most important data sets we use in our analysis are as follows (see also Fig. 1):

- Images from the Soft X-ray Telescope (SXT) onboard Yohkoh (Tsuneta et al. 1991). For the flare, partial-frame images (PFIs) from 08:58 to 09:24 UT are available in three filters (Be119, Al12 and AlMg) and in all SXT spatial resolutions. However, in the quarter and half resolution images (4.'9 and 9.' 8 per pixel, respectively) the flare is saturated, therefore we did not use these data. The set of images in the best, full resolution (2'. 45 per pixel) can be divided into two time intervals. Before 09:06 UT there are observations in three filters mentioned-above. After that moment, observations are only in the AlMg filter. Thus, the SXT diagnostic of the flare can be performed only for the first interval, between 08:58 and 09:06 UT. The full resolution SXT images were utilized to study the flare morphological evolution and to obtain geometrical, kinematic and physical parameters of the most interesting flare areas. The selected images were reduced using SolarSoftWare procedures. To estimate the emission measure $(E M)$ and temperature $(T)$ of flare plasma, we used the filter ratio method (FRM), as described by Hara et al. (1992).

- The measurement of hard X-ray (HXR) flux from the Hard X-ray Telescope onboard Yohkoh (Kosugi et al. 1991). These data cover the time interval from 08:56 to 09:24 UT. The 

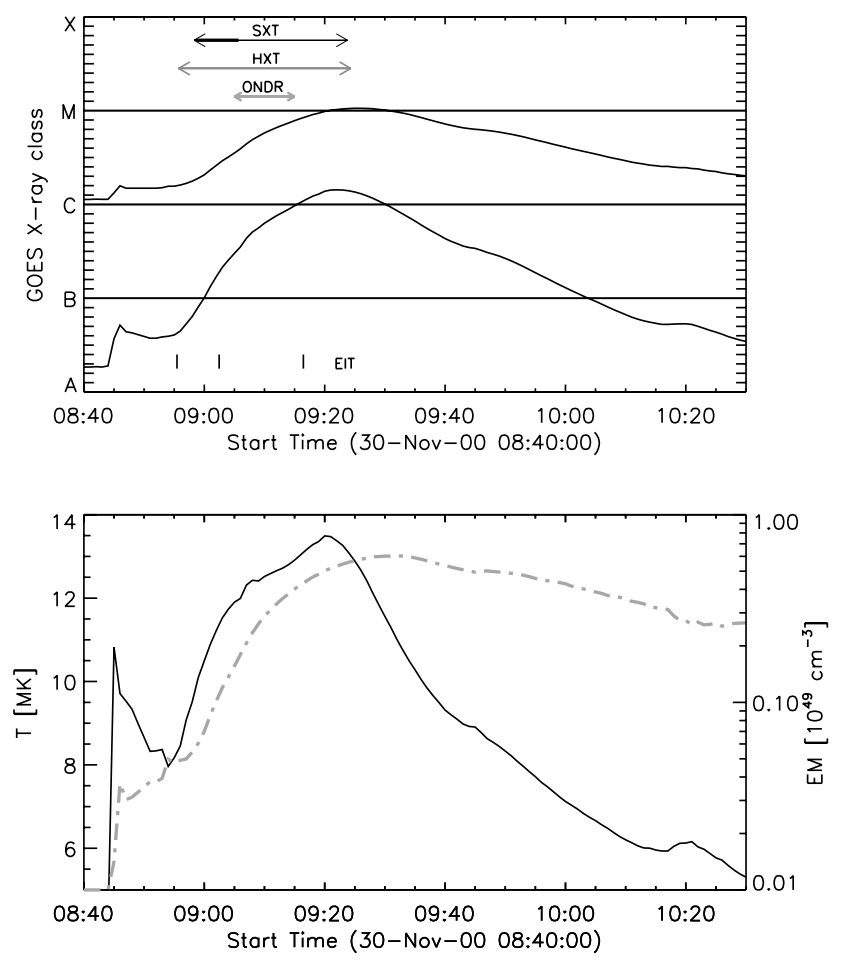

Fig. 1. The upper panel: GOES/SEM X-ray fluxes for the 30 November 2000 flare (upper curve: 1-8 $\AA$, lower curve: $0.5-4 \AA$ ). Arrows mark time intervals of available X-ray (SXT, HXT) and radio (ONDR) observations. The thicker part of the SXT arrow shows the time interval when the SXT diagnostic can be performed. The observation times for SOHO/EIT images shown in Fig. 5 are marked by vertical dashes. The lower panel: temperature (black line) and emission measure (grey dashdot line) for the flare calculated from GOES/SEM measurement.

time resolution of the data is 0.5 s before 09:06 UT and $4 \mathrm{~s}$ after that moment. For our analysis, we reduced the higher resolution in the first time interval to $4 \mathrm{~s}$. The HXT measurements were used to investigate temporal evolution of HXR emission and its correlation with the flare radio emission. In addition, we reconstructed images from HXT data to analyse the flare morphology in HXR. The images were synthesized using the maximum entropy method (MEM; Frieden 1972; Gull \& Daniell 1978; Willingale 1981; Sakao 1994), with modulation patterns computed by Sato (Sato 1997; Sato et al. 1999).

- The radio dynamic spectrum provided by the Ondřejov (ONDR) radiospectrograph (Jiřička et al. 1993). The spectrum covers the frequency range from 800 to $2000 \mathrm{MHz}$ and the time-interval from 09:05 to 09:15 UT. Temporal and frequency resolution is $0.1 \mathrm{~s}$ and $4.7 \mathrm{MHz}$, respectively. The ONDR spectrum was used to investigate temporal and frequency behaviour of the flare radio emission and its correlation with HXR emission.

In addition to the mentioned data, we also utilized the following observations:

- Images from the Extreme ultraviolet Imaging Telescope (EIT) onboard SOHO (Delaboudinière et al. 1995). For the analysed flare, EIT images in $195 \AA$ are available. Temporal resolution of the images is about $7 \mathrm{~min}$. We selected three of these images made at the beginning and during the rise phase of the flare. The images were used to study overall structure of the NOAA AR 9236, which enables us to construct a possible scenario of the flare formation.
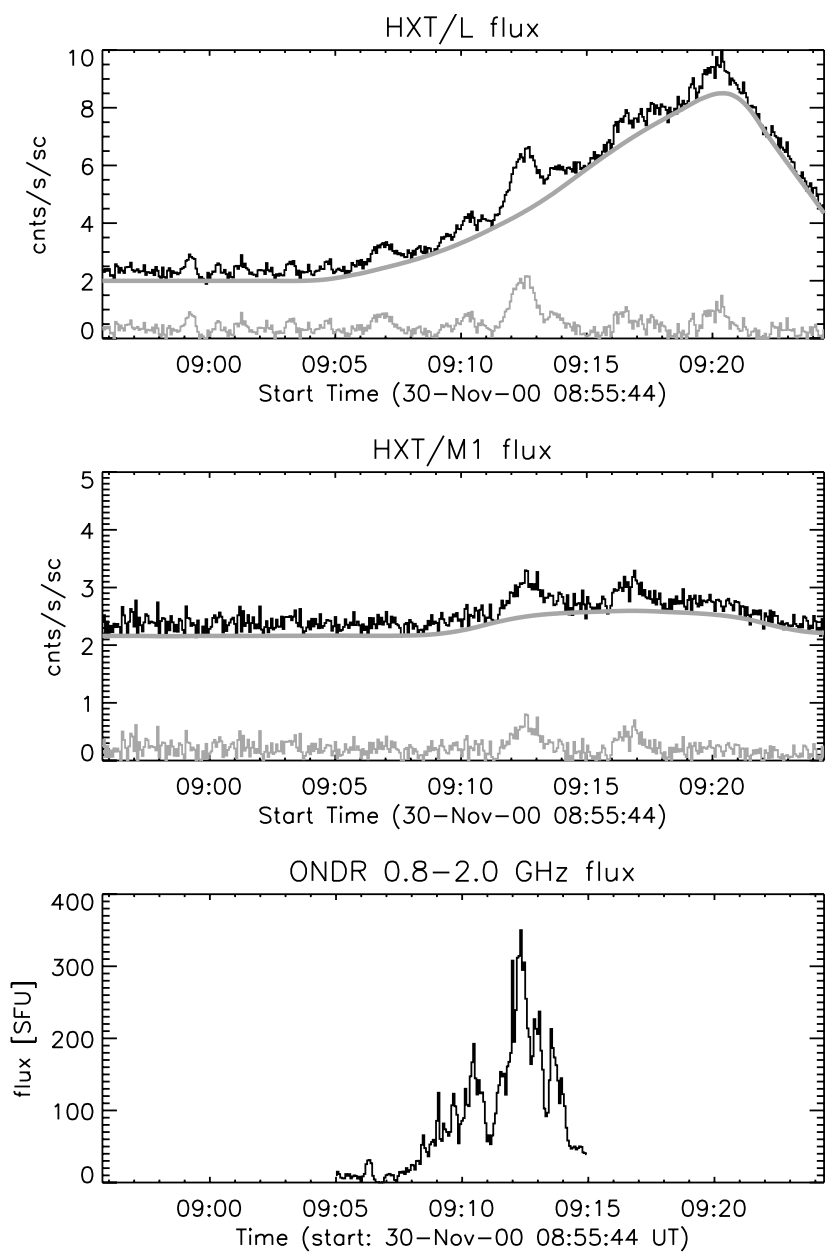

Fig. 2. Two upper panels: the HXR flux of the 30 November 2000 flare measured by Yohkoh/HXT in channels L and M1 (black lines). The flux was divided into two components: impulsive (thiner grey line) and smooth (thicker grey line). The impulsive component is shown after background subtraction. The lower panel: integrated radio flux recorded in ONDR in the range 800-2000 MHz.

- Solar X-ray light curves from the Space Environment Monitor (SEM) onboard GOES satellites (Donnelly et al. 1977). SEM provides flux measurements in two bands: 1-8 $\AA$ and $0.5-4 \AA$. Using these measurements, we estimated total emission measure and mean temperature of the flare using FRM.

\section{The analysis of observations}

The flare of 30 November 2000 occurred in NOAA AR 9236 at the location N17 W89. It began at 08:55 UT (see Fig. 1); earlier (at 08:46 UT) there was a weak X-ray flux maximum, although connection with the flare is unclear. The analysed flare had slow increase of brightness and reached the maximum (M1.0) at 09:26 UT. The flux then decreased for about $2.5 \mathrm{~h}$. Thus, the analysed flare belongs to the so-called long-duration events (LDE). From the ratio of signals in the two GOES/SEM channels, we calculated the mean temperature of the flare using Chianti 5.1 coronal abundances (see Fig. 1). The temperature increased from 8 (at 08:54 UT) to $13.5 \mathrm{MK}$ in about $26 \mathrm{~min}$. The increase was first steeper ( $4 \mathrm{MK}$ in $11 \mathrm{~min}$ ) then flatter (1.5 MK in $15 \mathrm{~min}$ ). The temperature maximum was reached at about 09:20 UT, and from that moment, there was monotonic 

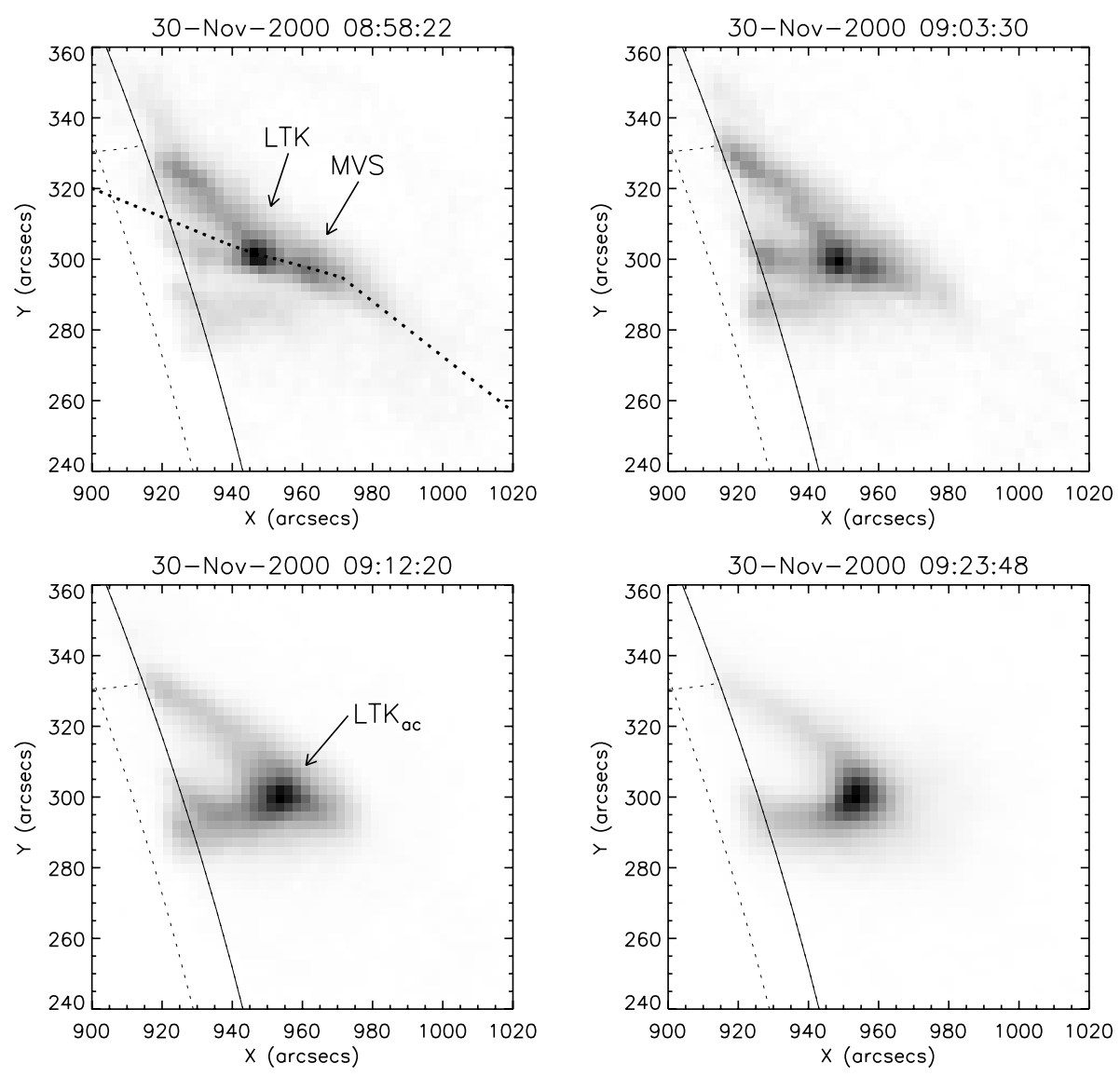

Fig. 3. The selected Yohkoh/SXT images of the 30 November 2000 flare made in the AlMg filter. The dotted line in the first image marks the path across the main parts of the flare. Over the path we estimated the physical parameters (see Fig. 10). The solar limb and heliographical grid are shown.

decrease of $T$. The time-profile of $T$ was asymmetric: increase just before maximum was flatter than decrease observed afterwards. We found that this behaviour was similar to the timeprofile of HXR (see below). Temporal changes of total emission measure of the flare were similar to flux changes. The maximum $E M$ was reached at about 09:30 UT.

HXR emission of the flare was recorded by HXT only in channels L (14-23 keV) and M1 (23-33 keV) and was weak (less then 10 counts/s/subcollimator; Fig. 2). In both channels, HXR emission was superposition of a smooth and impulsive component. To separate the components we fitted a polynomial to the total HXR flux time-profile. The polynomial was taken as an estimation of the smooth component. The impulsive component was obtained by subtracting the fitted polynomial from the total flux. The time-profile of the smooth component in channel $\mathrm{L}$ was very similar to the temperature time-profile estimated from SEM data. This suggest that the component was emitted by hot plasma. The ratio $R$ of the fluxes measured in the $\mathrm{L}$ and M1 channels (so-called hardness ratio) for this component was 0.03-0.14. Assuming thermal emission of plasma (singletemperature thermal Bremsstrahlung), the value of $R$ can be expressed in terms of temperature of emitting plasma. Using this assumption, we calculated that plasma emitting the smooth component was 20-30 MK hot.

The impulsive component was weaker than the smooth one. In channel M1 there were only two pulses recorded. More interesting is the time-profile of HXR in channel L, where some periodicity can be noticed. Before 09:04 UT there were five weak, short pulses, which occurred with a period equal to about $1 \mathrm{~min}$. After that moment, intervals between pulses became longer (2-4 min) and less periodic. The ratio $R$ for the impulsive component can be estimated for only two pulses recorded in both channels (at about 09:12.5 and 09:17 UT). The value of $R$ is $0.3-0.4$ and $0.5-0.7$ for the first and second pulse, respectively. Assuming that the impulsive component was emitted by non-thermal electrons (single-power-law non-thermal Bremsstrahlung), the obtained hardness ratio can be converted into the spectral index $(\gamma)$ of the power-law energy spectrum, equal to 5.3-6.0 and 3.8-4.8 for the first and second pulse, respectively.

The ONDR dynamic spectrum (see Fig. 7) shows so-called DPS (e.g. Karlický 2002) with limited frequency bandwidth and without significant global drift of the whole structure (the change of DPS global frequency during its duration was as small as $100-150 \mathrm{MHz}$, i.e., the global drift was only $\approx 0.4 \mathrm{MHz} / \mathrm{s})$. The first weak pulses were recorded just after 09:06 UT, but the main brightest DPS ranged from 09:08.5 to 09:14.2 UT. We integrated the observed radio flux over frequency and reduced temporal resolution to $4 \mathrm{~s}$ in order to compared it with the HXR impulsive component (see Fig. 2). The radio emission was more variable than the impulsive component, nevertheless, three pluses recorded in the L channel at 09:10:10, 09:12:10 and 09:13:20 UT (time of maximum) had counterparts in radio flux. This indicates that the impulsive component was emitted by non-thermal electrons in the close location as radio emission.

Figure 3 presents selected SXT images showing time evolution of the flare. In the first SXT images, we see the flaring loop with a bright source at the top (LTK - loop-top kernel). The loop had a distinct cusp-shape structure above the LTK. Within the structure there was another source, which moved downwards towards the LTK (MVS - moving source). As time passed, the distance between both sources decreased and finally the MVS 
Table 1. The differences in kinematics and morphology of the 20 November 2000 flare resulting from images made in the AlMg, Al12 and Be119 filters.

\begin{tabular}{cccccc}
\hline \hline Filter & $\begin{array}{c}\text { LTK - MVS distance } \\
\text { at 09:00 UT } \\
{\left[10^{9} \mathrm{~km}\right]}\end{array}$ & $\begin{array}{c}\text { LTK - MVS } \\
\text { relative velocity } \\
{\left[\mathrm{km} \mathrm{s}^{-1}\right]}\end{array}$ & $\begin{array}{c}\text { Brightness of the } \\
\text { depression at 08:58:20 UT } \\
\text { [fraction] }\end{array}$ & $\begin{array}{c}\text { The depression } \\
\text { disappearance }\end{array}$ & $\begin{array}{c}\text { The centroids } \\
\text { contact }^{c} \\
{[\mathrm{UT}]}\end{array}$ \\
\hline $\mathrm{Be} 119$ & $7.4 \pm 1.2$ & $-21.5 \pm 2.3$ & 0.71 & $09: 02: 20( \pm 30 \mathrm{~s})$ & $09: 05: 40( \pm 30 \mathrm{~s})$ \\
$\mathrm{Al12}$ & $8.8 \pm 1.2$ & $-22.6 \pm 2.3$ & 0.57 & $09: 03: 20( \pm 30 \mathrm{~s})$ & $09: 06: 30( \pm 30 \mathrm{~s})$ \\
$\mathrm{AlMg}$ & $9.8 \pm 1.2$ & $-20.0 \pm 1.6$ & 0.50 & $09: 03: 30( \pm 30 \mathrm{~s})$ & $09: 07: 40( \pm 60 \mathrm{~s})$ \\
\hline
\end{tabular}

${ }^{a}$ Brightness (relative to the brightest pixel) of the depression between LTK and MVS in the brightness profile over the path shown in Fig. 3.

${ }^{b}$ Moment of the depression between LTK and MVS disappearance.

${ }^{c}$ Moment of LTK and MVS centroids contact.
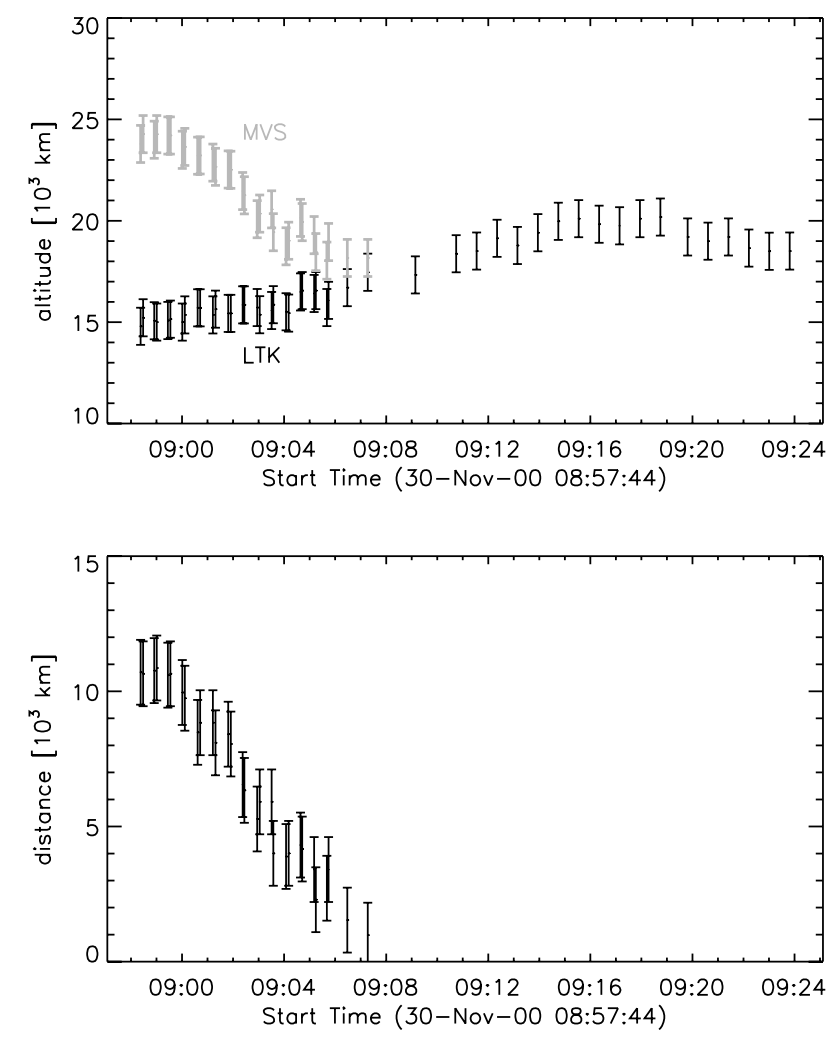

Fig. 4. The upper panel: the altitude (above the photosphere) of the LTK and MVS before the interaction and the LTK after the interaction. The altitude is corrected on the flare location. The lower panel: the distance between the LTK and MVS. The results were obtained from images made in the AlMg filter.

merged with the LTK (for LTK, after the merge with the MVS, we use the abbreviation LTK $_{a c}$ ). After the coalescence, the loop lost its cusp shape and the top of the loop became rounded.

To study the process of coalescing in detail, we estimated the altitudes $(h)$ of LTK and MVS and the distance $(d)$ between them in each available SXT image. The source altitude was calculated as the distance between its centroid and the photosphere. The obtained altitudes are the corrected altitudes (considering the flare position on the solar disc), not projected on the image plane. The results for the AlMg filter are shown in Fig. 4. The images made in this filter cover a longer time-interval than those made in the Al12 and Be119 filters and, this is why they give us more information on the kinematic evolution of the flare. The LTK showed typical upward motion. The velocity of the LTK motion had three stages: $2.5 \mathrm{~km} \mathrm{~s}^{-1}$ before the source's centroids contact, $6.5 \mathrm{~km} \mathrm{~s}^{-1}$ after that moment, until 09:16 UT when the LTK stopped at altitude $2.0 \times 10^{4} \mathrm{~km}$. The later decrease of altitude might not be real. During that time, the loop (and LTK) lost its cusp shape. This change could affect the determination of the centroid location. In the first SXT images of the flare, the MVS was almost at $2.5 \times 10^{4} \mathrm{~km}$ above the photosphere. Its altitude was systematically decreasing and at 09:07:40 ( $\pm 60 \mathrm{~s})$ the LTK and MVS centroids contacted. Relative velocity between these two sources was $-21.0 \pm 1.6 \mathrm{~km} \mathrm{~s}^{-1}$.

For the three filters, there were differences in the estimated altitudes, providing us with information regarding the thermal structure of both sources, and the area between them. To explain the differences, we must remember the SXT thermal sensitivity (response) depends on filter: the thicker the filter, the higher the sensitivity of SXT to more energetic photons and (in implication) to hotter plasma. From the three filters we used, the thinnest is the AlMg filter (maximum sensitivity for $<10 \mathrm{MK}$ ) and the thickest is Be119 (maximum for $>10 \mathrm{MK}$ ). Comparison of the images made in the three filters gives the following differences in kinematics and morphology of the flare (see also Table 1):

- The thicker the filter, the higher the altitude of the LTK centroid. This means that the LTK had vertical stratification of temperature: hotter plasma was located higher. Such a difference was not observed for the MVS, suggesting that in this source there was no significant vertical thermal stratification. See also the description of temperature maps below.

- Brightness of the depression between the LTK and the MVS (in the brightness profile over the path marked in Fig. 3, relative to the brightness of both sources) was higher in images made with thicker filters. This suggests that the depression contained relatively more hot plasma.

- The thicker the filter, the earlier the moment of the source's centroids contact. This difference results from the fact that the LTK centroid was observed higher in the thicker filter and consequently was closer to the MVS centroid (the relative velocity of the two sources approaching each other was similar in all three filters).

SOHO/EIT images can provide information about the overall magnetic structure of the NOAA AR 9236, where the flare occurred. In the EIT image made just before the flare (see the first image in Fig. 5), we see several, (4-6) $\times 10^{4} \mathrm{~km}$ high, loops forming the arcade. Seven minutes later, the LTK and MVS sources can be seen (see the second image in Fig. 5). Both sources had formed within one of the arcade loops. The cusp shape above the LTK, seen in the SXT images, extended along this loop. In the last of the selected EIT images, we see (as in the SXT images made at the same time) the LTK embedded in a distinct loop. From the the EIT images, a possible scenario of the flare formation can be inferred. It appears that two loops of the arcade interacted, forming the current sheet between them and here, due to the tearing mode instability, the LTK and MVS (plasmoid) were 

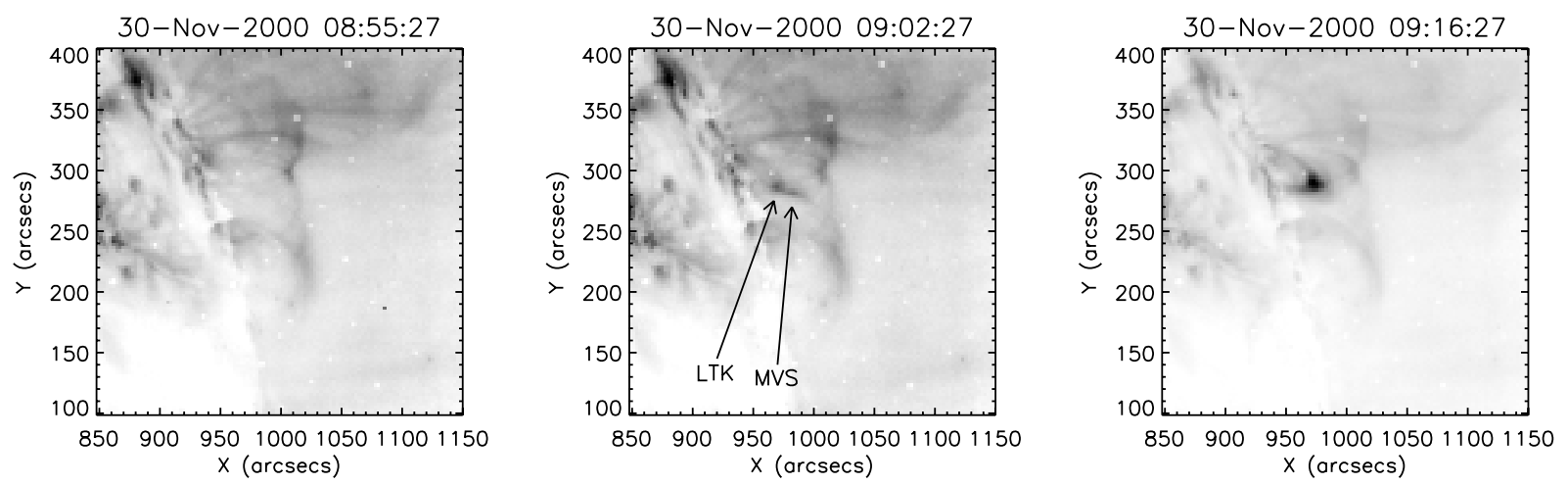

Fig. 5. The SOHO/EIT $195 \AA$ A images showing overall magnetic structure of the NOAA AR 9236 in which the analysed flare occurred. The first image was obtained just before the flare beginning. The second and third show the region before and after the LTK-MVS interaction, respectively.
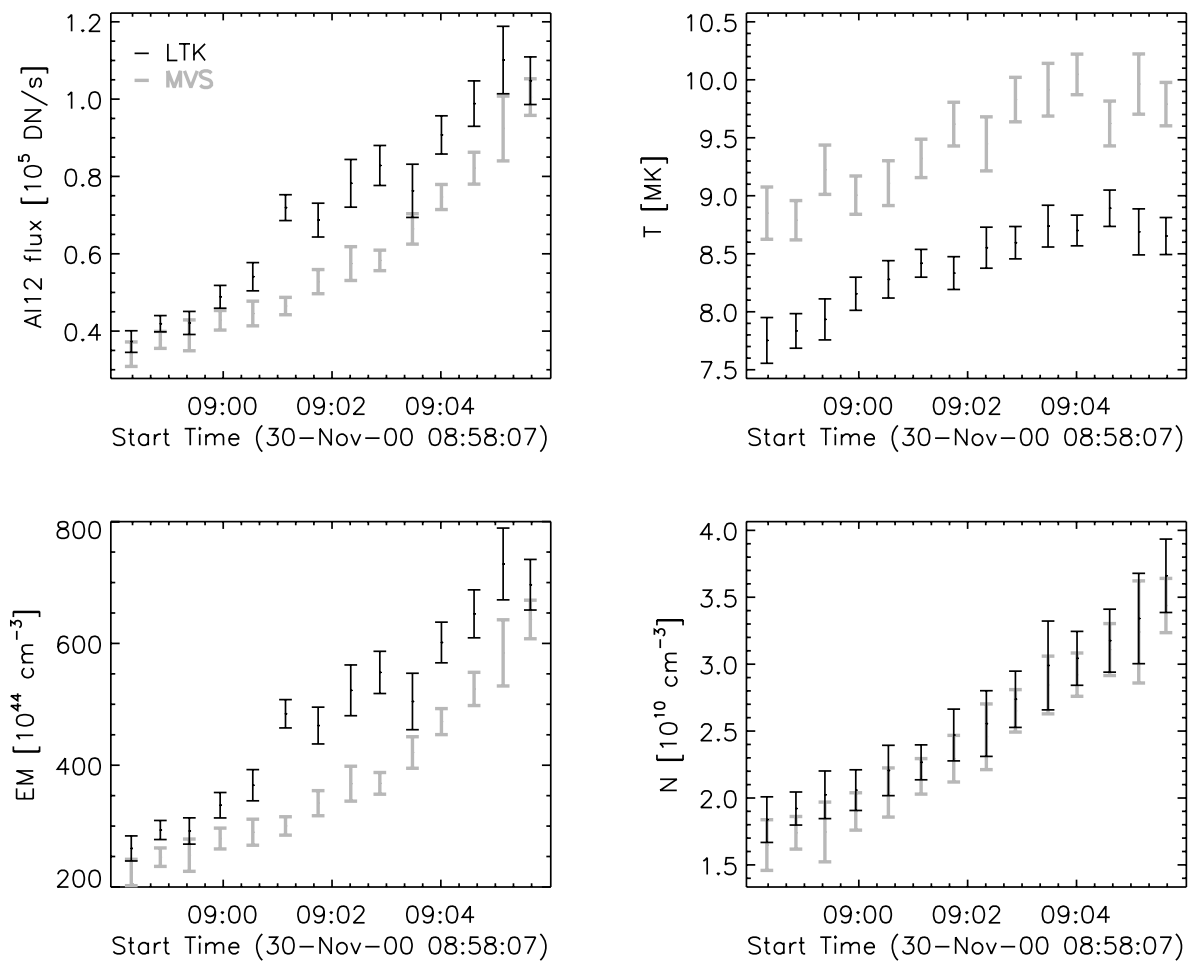

Fig. 6. Brightness, temperature, emission measure and density for the LTK and MSV, obtained from the $\mathrm{Be} / \mathrm{Al}$ diagnostic.

formed (see also the numerical modelling made by Bárta et al. 2007).

To estimate the physical parameters of both sources, we extracted in each Al12 image the area containing the sources. The boundary of the area was taken at an intensity isoline (isophote) 50 percent $\left(I_{50}\right)$ relative to the brightest pixel in any given Al12 image. We then divided the area into two subareas, each containing one of the sources. The division was made perpendicular to the line connecting the LTK and MVS. In the next step, in each Al12 and Be119 image, we summed the flux within these two subareas to estimate the mean $T$ and total $E M$ of plasma inside the LTK and MVS (Be/Al diagnostic). Using the obtained emission measure, we can calculate the mean electron density $(N)$ within each source from the simple equation: $N=\sqrt{E M / V}$, where $V$ is the volume of emitting plasma. However, to estimate $V$ we need to know the source dimension along the line-of-sight. This dimension cannot be determined directly from the SXT images, it must be reasonably estimated. We took, as this dimension, the width of each source defined by the isophote $I_{50}$ (in the direction perpendicular to the line connecting the LTK and MVS).
The results of the sources diagnostic are shown in Fig. 6 . The LTK was brighter than the MVS, as seen in the Al12 images, but in the Be119 images (thicker filter), both sources had a very similar flux. This means that the mean temperature of the MVS was higher than that of the LTK. This conclusion is confirmed by the $T$ estimation. Although both sources had similar time-variations of temperature, the MVS was about 1 MK hotter than the LTK. Time-variations of the $E M$ of both sources were also similar, but the LTK had emission measure that were 1.1-1.5 times larger. The mean density of the sources was almost the same in value and time-profile. Similar temporal variations of all estimated physical parameters suggest that the LTK and MVS were not separate sources; they were physically (magnetically) connected.

The observed interaction and coalescence of the LTK and MVS should be similar to coalescence of two plasmoids. As shown by Karlický \& Bárta (2007), such a process leads to efficient electron acceleration and to radio emission (plasma waves mechanism) in the form of a pulsating structure with a limited frequency bandwidth. If, in addition, there is a change of density of the coalescing plasmoids, due to e.g. their upward or 


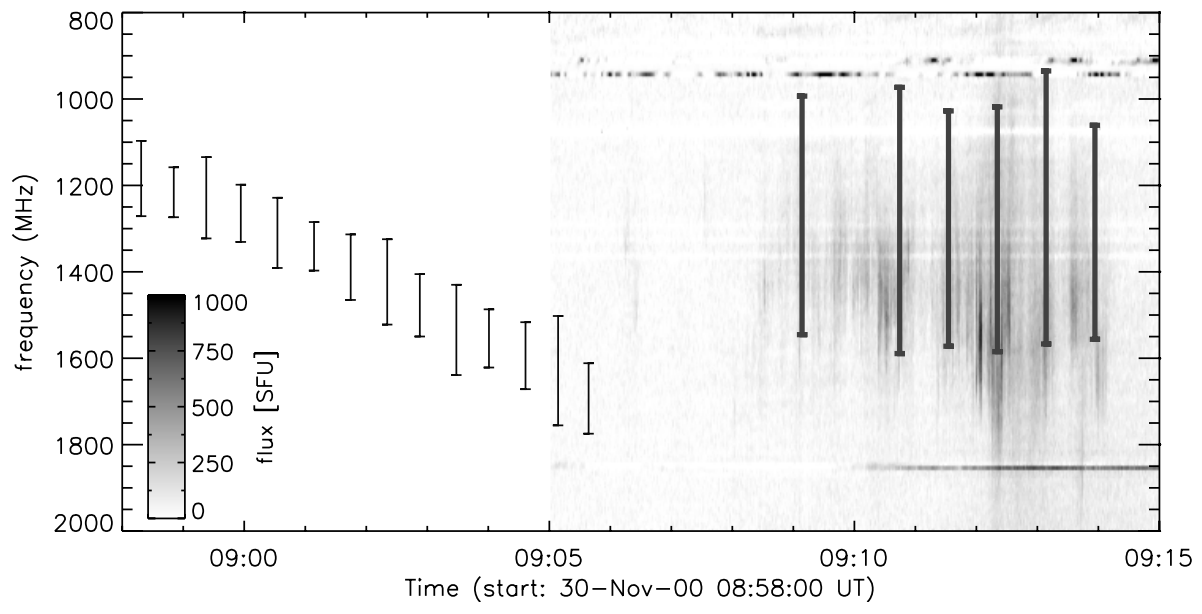

Fig. 7. The ONDR dynamic spectrum for the analysed flare compared with predictions. a) The thin vertical bars show predicted frequency (with errors) of radio emission from the interacting X-ray sources. The frequency was calculated from the mean density of the LTK+MVS, assuming fundamental plasma frequency band. b) The thick vertical bars show the predicted frequency of radio emission from the $S_{\text {DPS. }}$. The frequency at each moment was calculated from the minimal and maximal density within the $\mathrm{S}_{\mathrm{DPS}}$, assuming fundamental plasma frequency band.

downward motion, the emitted pulsating structure will show a global frequency drift (negative or positive).

To verify whether the observed DPS might be produced by the LTK-MVS interaction, we calculated the frequency of radio emission, using the density estimated above, as: $f_{p}=$ $9000 N^{1 / 2}$ [Hz]. The results are shown in Fig. 7. Unfortunately, the SXT diagnostic ends before the first DPS pulse, however, by extrapolating the estimated frequencies (assuming constant drift, the flux increase measured in the AlMg images indicates that the density was still increasing after 09:06 UT), we see that the predicted $f_{p}$ are higher than observed values by factor $\approx 1.2-1.4$. However, as shown by Karlický \& Bárta (2007), the pulsating emission is mainly produced in the external (i.e. less dense) parts of interacting plasmoids. For the observed and predicted frequencies to agree, the observed density should be lower, by a factor $\approx 1.4-2.0$, which is a reasonable value. The second disagreement between the observed and predicted frequencies is that the observed DPS showed no substantial global drift towards higher frequencies, as the predicted emission. However, this disagreement can be explained if we assume that the drift of a DPS (or the lack of the drift) is caused by the source altitude change. At the time of the DPS observation, the plasmoid had practically stopped due to the interaction with the LTK, possibly leading to no significant global drift of the emitted DPS.

There is another possible explanation for the observed DPS; it might be produced by electrons, which after acceleration during the LTK-MVS interaction, escaped from the coalescent sources to an other site(s) (Karlický \& Bárta 2007). From the observed characteristics of the DPS, we can conclude that it was emitted from the region with: (a) a lower density than in the interacting sources, and (b) a nearly constant density at least during the time of the DPS (09:08.5-09:14.2 UT). The main difficulty in finding such an area is the lack of diagnostic after 09:06 UT. During the time of the DPS we only have the AlMg images. Fortunately, the SXT had higher sensitivity to density variations than to temperature variations, at least in the typical flare temperature range. In the case of the AlMg filter, the signal measured by the SXT (expressed in so-called $D N$ - data number) changed by only a factor of 1.4 in $T$ range 5-25 MK (see Fig. 9 in Tsuneta et al. 1991), while the $D N$ is proportional to $N^{2}$. Using this characteristic, we can roughly estimate from the AlMg images the relative changes of density, or even the absolute value of $N$ by extrapolating the results from the $\mathrm{Be} / \mathrm{Al}$ diagnostic.

Assuming that all of the observed variations of brightness in the AIMg images were caused by density variations, we carefully analysed the SXT data to find the site from which the DPS

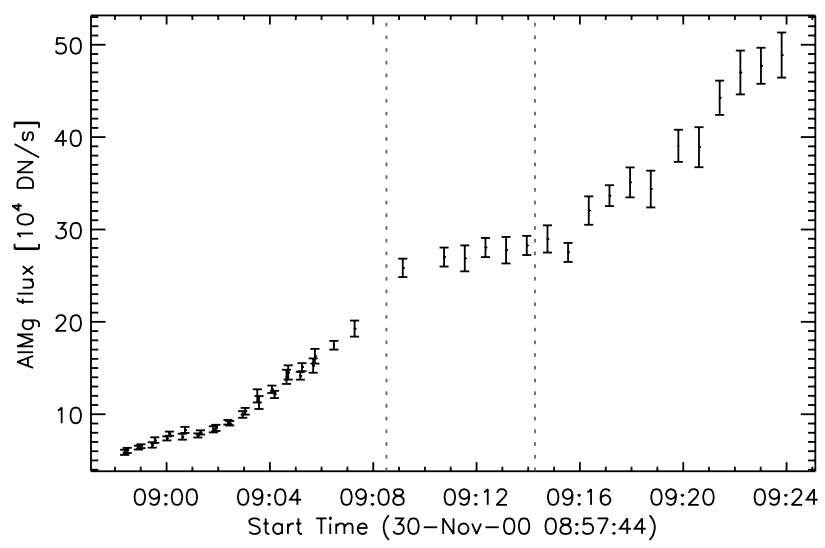

Fig. 8. Time-variations of brightness in the $S_{\text {DPS. }}$ The vertical dashed lines mark the radio DPS time interval.

could be emitted, i.e., the site where, at least during the radio emission, there was (almost) no significant change of brightness. From ONDR dynamic spectra we see that the DPS global drift was about $0.4 \mathrm{MHz} / \mathrm{s}$ (the DPS frequency only changed by $100-150 \mathrm{MHz}$ during the DPS duration). Using the relations between $f_{p}, N$ and $D N$, we can state that to obtain such a small change of frequency, the brightness of the area emitting the DPS should not increase by more than about 1.4 times during that time. Thus, we searched for areas where (during the DPS) the brightness increased by no more than 1.4 times.

We found two such areas. The first was a large area limited by the flare loop (below the LTK) and can be rejected because it was not connected with the flare. The second site was located above the $\mathrm{LTK}_{a c}$, partially overlapping with the HXR source. As previously mentioned, since time-variations of the radio emission were similar to the HXR radiation, we can suppose that the DPS was emitted from the same location as the HXR flux, i.e., from above the $\mathrm{LTK}_{a c}$ (see HXT images in Fig. 11). Thus, for further analysis, we only took the part of the brightness smallvariation site that was in common with the HXR source $\left(S_{D P S}\right.$; see the second image in Fig. 11). We determined the brightness time-variations for the $S_{\text {DPS }}$ region. The result is shown in Fig. 8. As one can see, during the DPS, there was a distinct plateau in the time-profile of the $S_{\text {DPS }}$ brightness. To confirm that the $\mathrm{S}_{\mathrm{DPS}}$ was the site where the DPS was produced, we tried to estimate the density in this region and then transform $N$ to radio frequency to compare with the DPS frequency. For each pixel of the $S_{\text {DPS }}$, we took the density estimated from the last available pair of Be119/A112 images (at 09:05:38 UT) and the AlMg 

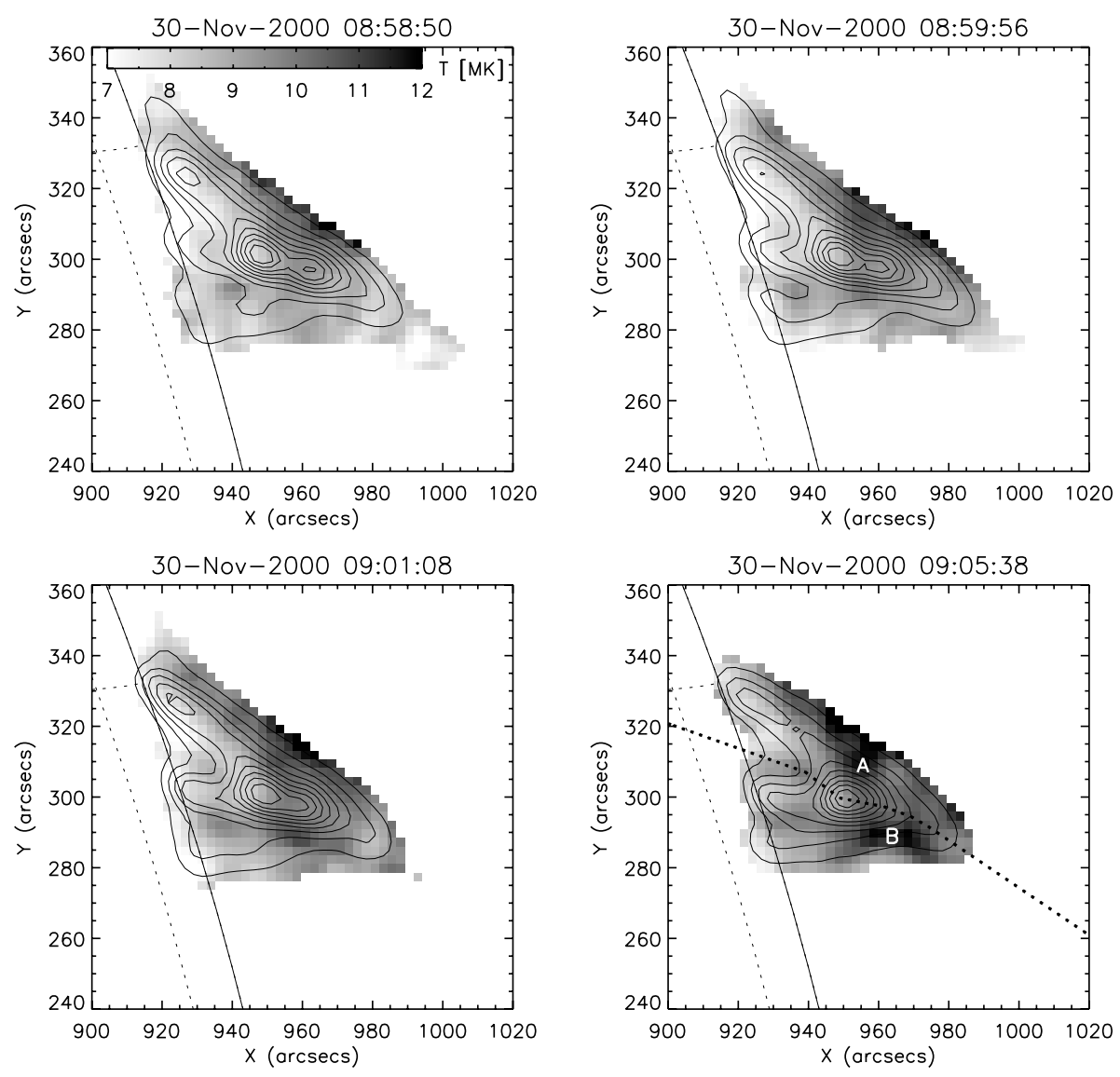

Fig. 9. The selected temperature maps obtained from the $\mathrm{Be} / \mathrm{Al}$ diagnostic. The brightness distribution from the Al12 images is overlaid as isolines. The contours have values from 0.1 to 0.9 (with increment 0.1 ) relative to the brightest pixel in a given A112 image. The solar limb and heliographical grid are shown.

brightness at that time, and using the relation $D N \propto N^{2}$, we calculated the density in these pixels during the DPS time. We then transformed $N$ to plasma frequency and compared the result with the DPS frequency (see Fig. 7). The predicted frequency and bandwidth are in good agreement with the DPS characteristics, thus, we can conclude with high probability that the $S_{\text {DPS }}$ was the source of the DPS.

The interaction between the LTK and MVS should also be revealed in the thermal structure of the flare, therefore we analysed the temperature distribution on $T$ maps obtained from the $\mathrm{Be} / \mathrm{Al}$ diagnostic (see Fig. 9). Firstly, there was a difference in the thermal structures of the LTK and MVS. The first source had a vertical temperature distribution, i.e., its lower part was cooler than the upper part. The MVS was not only hotter than the LTK, but there was also no such vertical stratification of $T$. Since the thermal conduction is most efficient along magnetic field lines, the observed thermal structure provides us with information about the magnetic structure of both sources. The structure of the LTK can be explained by the model of the collapsing magnetic trap (e.g. Somov \& Kosugi 1994; Jakimiec 2002; Karlický \& Bárta 2006). The upper part of the LTK was closer to the reconnection site; it should be hotter because it was "made of" newly formed magnetic traps and the most recently heated plasma. The lower part of the LTK was older, built of magnetic traps formed earlier and plasma within them that had time to cool down. The thermal structure is also in agreement with the common observational fact that very hot plasma is usually present above loop-top sources, while cooler UV loops are below them. On the other hand, if the MVS is a plasmoid, it should have closed, circular magnetic field lines, allowing a temperature balance between its lower and upper parts. Moreover the MVS, according to our scenario of the flare formation, was

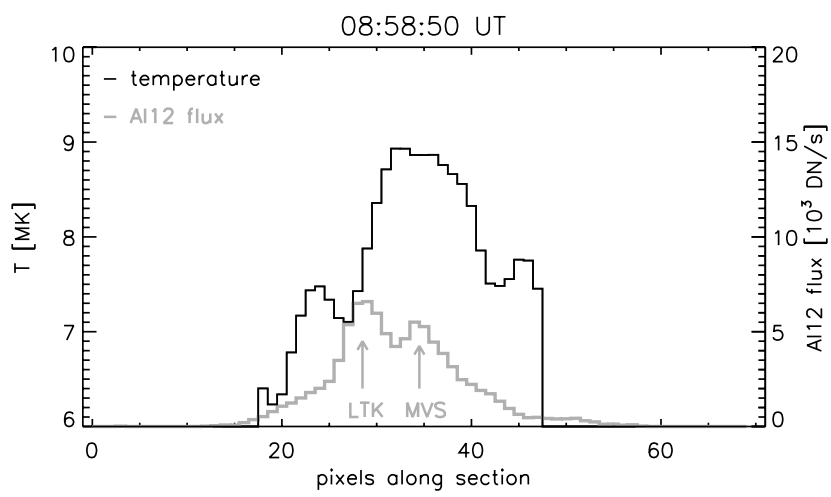

Fig. 10. Temperature (black line) and brightness (grey line) profiles estimated for the flare before the LKT and MVS coalescence. The profiles were obtained over the path shown in Figs. 3 and 9. Temperature was not calculated for pixels with numbers less than 18 and greater than 47 because low signals within these pixels might give unreliable results.

heated simultaneously from below (reconnection with the LTK) and from above (main reconnecting current sheet between the arcade loops; see Fig. 13).

The most interesting observation is the distribution of hot areas. In the thermal maps, there were two hot regions on opposite sides of the interacting sources (areas A and B in Fig. 9). The areas formed near the brightness depression between the sources, and as time passed they became hotter. Moreover, before the sources coalescence, the highest temperature along the path shown in Figs. 3 and 9 was observed in this brightness depression (see Fig. 10). The interaction between the LTK and MVS led to local plasma heating near the interaction site. Heated 

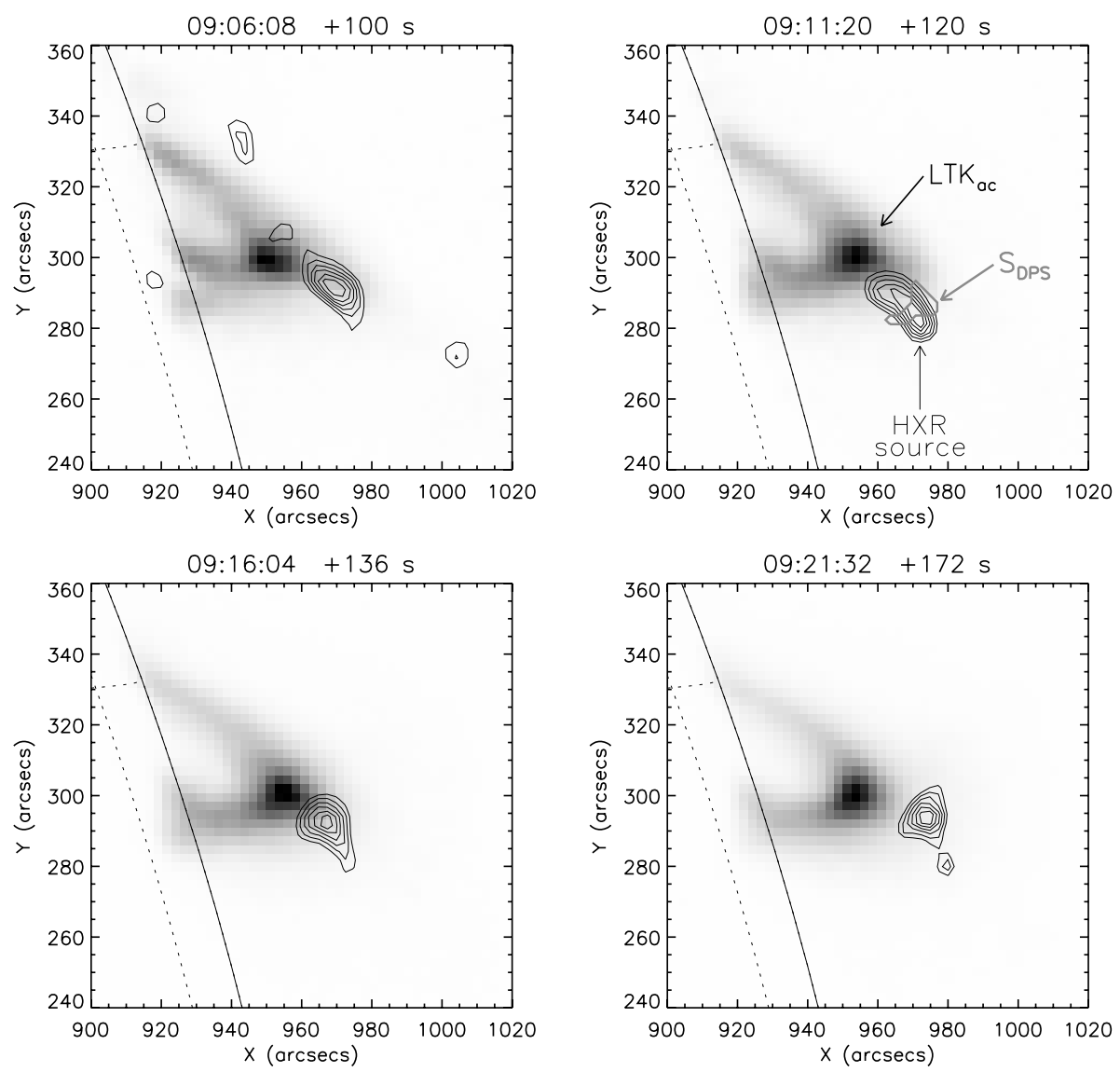

Fig. 11. The selected HXT/L images overlaid as contours (thin lines) on corresponding $\mathrm{AlMg}$ images. The contours have values from 0.4 to 0.9 (with increment 0.1 ) relative to the brightest pixel in a given HXT/L image. For each HXT/L image, the starting time of integration and its duration are given. The thick contour in the upper-right image shows the area $S_{\text {DPS. }}$ The solar limb and heliographical grid are shown.

plasma may then have been "squeezed out" of the site, forming the hot areas A and B.

Only in channel L was the number of counts sufficient to obtain HXT images for the analysed flare. Figure 11 shows a sequence of selected HXT images overlaid on AlMg images. In all obtained images there is only one HXR source, just above the $\mathrm{LTK}_{a c}$. Its position was almost stable; there was no systematic displacement and shifts were smaller than 10 arcsec. The position was in good agreement with the position of the hot area B (compare the last image in Fig. 9 with the first image in Fig. 11), thus the HXR source (the smooth component) was probably formed by very hot plasma "squeezed out" of the LTK-MVS interaction site or/and heated by electrons accelerated in the site. Keeping in mind that the radio DPS was also emitted from that location, we can conclude that the impulsive/nonthermal HXR component was also emitted by particles, which, after acceleration, escaped from the LTK-MVS contact site.

\section{Discussion and conclusions}

In this paper, we study the unique observations of the 30 November 2000 flare, in which an interaction and coalescence of the loop-top kernel with the plasmoid were recorded. Such a process was predicted by some simulations (e.g. Bárta et al. 2007; Riley et al. 2007). Moreover, Karlický \& Bárta (2007) showed that interaction and coalescence of two plasmoids leads to effective particle acceleration and plasma heating. The most energetic particles are located in the external parts of the plasmoids and some of them can even escape outside. Although a loop-top kernel can have a different internal magnetic structure than a plasmoid, interaction and coalescence of a loop-top kernel with a plasmoid should be similar to the interaction of two plasmoids. Thus, the increase of X-ray and radio (DPS structure) emissions during the event and the increase of the temperature in the region between the LKT and the MVS are considered here as evidence that the described event was a real interaction process.

The very beginning of the flare was not well observed. However, the formation of the LTK and MVS can be inferred from the EIT images and known numerical simulations (e.g. Bárta et al. 2007). The flare started due to an interaction of two loops in the loop arcade. Between these two loops the current sheet was formed and, due to the tearing-mode instability and loops fixed in the photosphere, the growing flare loop and plasmoid were formed. The tension force in the inverted V-shape magnetic field structure around the plasmoid accelerated the plasmoid (MVS) downwards, and then after some time it interacted with the growing loop, having the X-ray kernel (LTK) at its top. The contact between the sources resulted in reconnection of their magnetic fields, particle acceleration and plasma heating. In the observations these processes manifested themselves as follows:

- During the LTK-MVS interaction two hot areas A and B developed on opposite sides of both sources, near the contact site. Moreover, in this site, between the sources, plasma was hotter than inside them. The hot plasma was also located around the MVS.

- At the end, and just after the sources coalescence, 20-30 MK hot plasma appeared in the area B.

- At the same time nonthermal electrons, which escaped from the LTK-MVS interaction site, emitted impulsive radio and $\mathrm{HXR}$ radiation. 


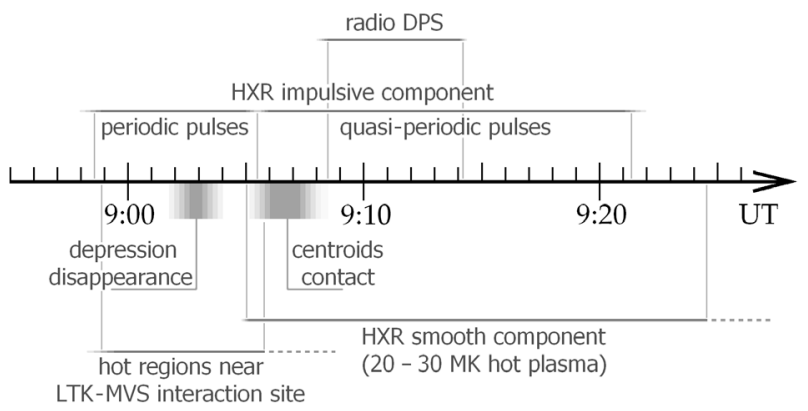

Fig. 12. Schematic drawing showing the time order of the most important observed phenomena.

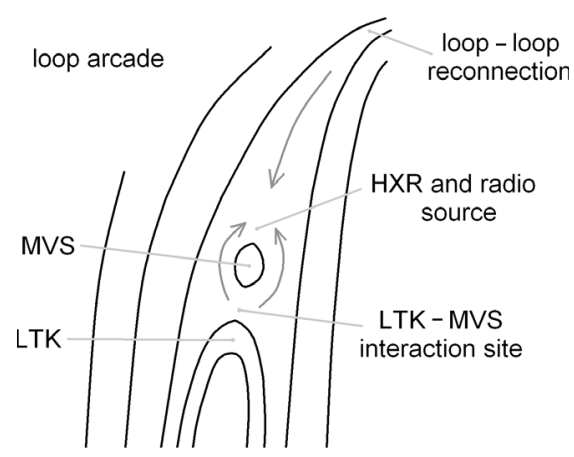

Fig. 13. Flare scenario. The arrows represent fluxes of accelerated electrons and heated plasmas.

- Periodic HXR pulses were detected during the interaction, and quasi-periodic pulses at the end and after the coalescence.

The location of hot plasma areas observed by the SXT and HXT and the (same) location of the source of impulsive emission in radio and hard X-rays, suggests that they might be formed by electrons, which, after acceleration, escaped from the LTK-MVS interaction site. The coalescence may also have "squeezed out" heated plasma outwards.

In summary (see also Fig. 12), the scenario of the processes under study are shown in Fig. 13. In the space between the two arcade loops, the flare loop with the LTK and MVS was formed and then the sources interacted. Note that the main reconnecting current sheet is between the arcade loops. It differs from the vertical current sheet below the rising magnetic rope, as assumed in the standard model of two-ribbon flares (Priest $\&$ Forbes 2000). In this scenario we have two reconnection sites: the reconnection between two arcade loops and the reconnection between the interacting LTK and MVS. Both accelerated the particles and heated the plasma (the particles and plasma flows are shown in Fig. 13). These particles and hot plasma accumulated just above the coalescent LTK-MVS where they produced the HXR and DPS radio emissions.

Acknowledgements. Authors thank Yohkoh, GOES and SOHO teems for excellent observations. This research was supported by Grant IAA300030701 of the Academy of Sciences of the Czech Republic and by Grant N203 193733 of the Polish Ministry of Science and High Education.

\section{References}

Acton, L. W., Feldman, U., Bruner, M. E., et al. 1994, PASJ, 44, L71 Bárta, M., Karlický, M., Vršnak, B., \& Goossens, M. 2007, CEAB, 31, 165 Chen, P. F., Shibata, K., Brooks, D. H., \& Isobe, H. 2004, ApJ, 602, L61 Delaboudinière, J.-P., Artzner, G. E., Brunaud, J., et al. 1995, Sol. Phys., 162, 291

Donnelly, R. F., Grubb, R. N., \& Cowley, F. C. 1977, NOAA Tech. Memo. ERL SEL-48

Frieden, B. R. 1972, J. Opt. Soc. Am., 62, 511

Gull, S. F., \& Daniell, G. J. 1978, Nature, 272, 686

Hara, H., Tsuneta, S., Lemen, J. R., Acton, L. W., \& McTiernan, J. M. 1992, PASJ, 44, L135

Jakimiec, J. 2002, ESA SP-506, 645

Jiřička, K., Karlický, M., Kepka, O., \& Tlamicha, A. 1993, Sol. Phys., 147, 203

Karlický, M. 2004, A\&A, 417, 325

Karlický, M., \& Bárta, M. 2006, ApJ, 647, 1472

Karlický, M., \& Bárta, M. 2007, A\&A, 464, 735

Karlický, M., \& Kosugi, T. 2004, A\&A, 419, 1159

Karlický, M., Fárník, F., \& Mészárosová, H. 2002, A\&A, 395, 677

Khan, M., Vilmer, N., Saint-Hilaire, P., \& Benz, A.O. 2002, A\&A, 388, 363

Kliem, B., Karlický, M., \& Benz, A. O. 2000, A\&A, 360, 715

Kosugi, T., Masuda, S., Makishima, K., et al. 1991, Sol. Phys., 136, 17

Krucker, S., Hurford, G. J., \& Lin, R. P. 2003, ApJ, 595, L103

Lin, R. P., Krucker, S., Hurford, G. J., et al. 2003, ApJ, 595, L69

Magara, T., Mineshige, S., Yokoyama, T., \& Shibata, K. 1996, ApJ, 466, 1054

Masuda, S., Kosugi, T., Hara, H., Tsuneta, S., \& Ogawara, Y. 1994, Nature, 371, 495

Ohyama, M., \& Shibata, K. 1998, ApJ, 499, 934

Priest, E. R., \& Forbes, T. 2000, Magnetic Reconnection: MHD Theory and Applications (Cambridge, UK: Cambridge Univ. Press)

Riley, P., Lionello, R., Mikić, Z., et al. 2007, ApJ, 655, 591

Sakao, T. 1994, Ph.D. Thesis, Dept. of Astronomy, University of Tokyo

Sato, J. 1997, Ph.D. Thesis, Graduate Univ. of Advanced Studies, Tokyo

Sato, J., Kosugi, T., \& Makishima, K. 1999, PASJ, 51, 127

Shibata, K. 1999, Ap\&SS, 264, 129

Shibata, K., \& Tanuma, S. 2001, Earth Planets Space, 53, 473

Somov, B. V., \& Kosugi, T. 1997, ApJ, 485, 859

Tsuneta, S., Acton, L., Bruner, M., et al. 1991, Sol. Phys., 136, 37

Tsuneta, S., Hara, H., Shimizu, T., et al. 1992, PASJ, 44, L63

Willingale, R. 1981, MNRAS, 194, 359

Yokoyama, T., Akita, K., Morimoto, T., Inoue, K., \& Newmark, J. 2001, ApJ, 546, L69 